\title{
JURISPRUDENCIA AMBIENTAL EN ASTURIAS (SEGUNDO SEMESTRE 2017)
}

\author{
Alejandra Boto Álvarez \\ Profesora Contratada Doctora (acreditada PTU) de Derecho Administrativo \\ Universidad de Oviedo
}


Sumario: 1. Introducción: los vínculos recurrentes entre la actualidad administrativa y judicial, y dentro de ella. 2. Acotamiento al pastoreo de terrenos incendiados. 3. Conflictos de interés en la gestión del lobo: más cuestiones formales que materiales. 4. Otro episodio judicial en torno al nuevo-viejo Plan Estratégico de Residuos. 5. Tributos, medio ambiente y redes de telecomunicaciones.

\section{Introducción: los vínculos recurrentes entre la actualidad administrativa y la judicial, y dentro de ella}

Empieza a ser habitual en estas crónicas de jurisprudencia ambiental la aparición de los mismos asuntos que protagonizan la sección sobre normativa y política asturiana en la materia; a ello se une la constatación de la existencia de ciertos temas que parecen ya auténticos "clásicos" pero que siguen planteándose ante el Tribunal Superior de Justicia del Principado.

Así, sólo por citar un ejemplo, en el número precedente de esta revista, el asunto del acotamiento temporal al pastoreo de los montes incendiados como medida de adopción obligatoria para la regeneración de la cubierta vegetal quemada fue objeto de estudio tanto desde la perspectiva jurisprudencial como normativa. La materia sigue siendo de interés, y en la presente entrega ocurrirá otro tanto. Lo mismo va a suceder respecto a la gestión del lobo y, de nuevo, con el Plan Estratégico de Residuos.

Por otro lado, entre los asuntos de tratamiento cíclico en la jurisprudencia, la tributación "verde" ha vuelto a tener eco asimismo en el período que cubre esta crónica, por lo que procede volver sobre ella.

\section{Acotamiento al pastoreo de terrenos incendiados}

En la anterior entrega de esta sección se comentó la sentencia 45/2017, de 30 de enero, del Tribunal Superior de Justicia de Asturias, dictada en el recurso $892 / 2015$.

Con tal recurso se impugnaba una resolución de 13 de agosto de 2015 de la Consejería de Desarrollo Rural y Recursos Naturales que acotaba al pastoreo un monte incendiado. La parte recurrente pretendía la nulidad de la resolución por caducidad del expediente y arbitrariedad en la determinación del plazo de duración del acotamiento; subsidiariamente, en el caso de no considerar la 
nulidad íntegra de la misma, se solicitaba que se condenase al acotamiento al pastoreo computando el plazo desde la fecha del incendio.

Entonces ya se comentó que en el debate sobre la caducidad se habían intercambiado opiniones diversas respecto a si el plazo para resolver debía computarse desde la fecha en que las brigadas de investigación de incendios forestales del Principado habían tenido conocimiento del incendio (30 de octubre de 2014) o desde que esta información llegaba a la Consejería competente en materia forestal (13 de marzo de 2015, por tanto, ya con el incendio más que extinguido). En aquella ocasión el Tribunal justificaba la dilación de cinco meses en atención a que el servicio de prevención de incendios del Principado de Asturias, que es quien canaliza los datos e informes de investigación de causas de incendios forestales, no está adscrito a la Consejería que dicta el acto recurrido. Se argumentaba también que apreciar caducidad procedimental resultaría contrario al interés público y a los principios de economía y seguridad jurídica. Finalmente, el Tribunal estimó el recurso interpuesto por razones que tenían que ver con una incorrecta tipificación de la zona quemada como superficie forestal, sin pronunciarse sobre la cuestión de los plazos.

Este aspecto ha sido objeto de atención de nuevo, en las sentencias 385/2017 y 390/2017, ambas de 9 de mayo, que resuelven los recursos 890/2015 y 891/2015, respectivamente. El Ayuntamiento recurrente común había formulado pretensiones coincidentes con las de la sentencia cuyo comentario acaba de ser recordado. El Tribunal afirma de manera expresa que no cabe apreciar caducidad automática con los mismos argumentos que ya se habían invocado en la sentencia precedente. Descartado después que concurriese alguna causa de nulidad en la resolución (en particular se analiza, como en el fallo ya comentado, la falta de motivación, y una posible ilegalidad o arbitrariedad, que no se aprecian en los casos de autos), se desestiman los recursos con expresa imposición de costas, que se limitan en ambos casos a 500 euros.

Lo peculiar de estas sentencias, a mi entender, es que a pesar de estar incluido entre las pretensiones del recurrente como petitum subsidiario, el Tribunal no entra a dilucidar el momento desde el cual debería computarse el 
plazo de acotamiento (si desde el momento en que se dictó la resolución administrativa o desde la fecha el incendio). Se trata de todas formas de un aspecto que de manera sobrevenida ha dejado de tener relevancia, dado que todos los acotamientos por razón de incendio han sido levantados y dejados sin efecto según lo que se ha comentado en la sección de política y legislación de este mismo número de la revista.

\section{Conflictos de interés en la gestión del lobo: más cuestiones formales que materiales}

En su momento, distintas asociaciones conservacionistas impugnaron por pretendidos defectos procedimentales invalidantes el II Plan de Gestión del Lobo del Principado de Asturias (Decreto 23/2015, de 25 de marzo) y el Programa Anual de Actuaciones de Control del Lobo 2015-2016.

El primer recurso (505/2015) ha sido desestimado por Sentencia 286/2017, de 3 de abril. De entre los fundamentos de la impugnación', quizás los que más enjundia presentan desde la perspectiva ambiental sean el que pretendía la nulidad del Decreto por no haber sido sometido a evaluación ambiental estratégica, en cuanto que se trata de un plan sobre una especie protegida por el Convenio de Berna, sobre la que se aplican medidas de eliminación, y que se encuentra además presente en buena parte de la Red Natura 2000; o el que sostenía que las medidas de control de la especie previstas por el plan eran arbitrarias e inadecuadas desde el punto de vista del Convenio precitado y la Directiva hábitats, además de no estar suficientemente coordinadas con otras instancias a pesar de afectar a la población del lobo en España.

\footnotetext{
${ }^{1}$ Las pretensiones se sustentaban además en una pretendida falta de justificación de la propuesta o de su incidencia en el marco normativo, que el Tribunal descarta a la vista de toda la documentación obrante en el expediente y que habían cubierto, entre otras cosas, la evaluación de la eficacia del I Plan, con elaboración de sucesivas propuestas, informes y análisis de impacto. Esta dispersión se justifica atendiendo a que "difícilmente puede existir un solo documento que valore los aspectos de evaluación de la población a través de programas de monitoreo y de los registros de actuaciones cuya evaluación ha sido plasmada en los programas de actuaciones propuestas cada año" (Fundamento de Derecho segundo). Se había alegado también falta de competencia del órgano tramitador, por haberse anulado el nombramiento de la persona que dirigía la Dirección General encargada de la incoación y tramitación del expediente de revisión del Plan de Gestión del Lobo, y por una inadecuada catalogación de la especie animal. El Tribunal entiende que tales irregularidades no son invalidantes, por un lado porque existían suficientes informes técnicos del Servicio de Caza y Pesca y del personal técnico de la Dirección General, y por otro porque el error en la catalogación sólo tuvo incidencia en la distribución interna de competencias entre los servicios de la Dirección General, pero nunca estuvo en cuestión la falta de cualificación profesional de quien realizaba los informes (Fundamento de Derecho tercero).
} 
El Tribunal no acepta el criterio de que el Decreto tuviera que ser objeto de evaluación ambiental por la incorporación del término "plan" o porque se ha elaborado por una Administración pública debido a los posibles efectos negativos para el medio ambiente a valorar por el órgano ambiental,

en un caso, por su sentido exclusivamente conceptual y porque esa incidencia se asocia legalmente a proyectos en las materias relacionadas con la explotación de recursos naturales, ocupación de dominio público, telecomunicaciones, turismo, ordenación del territorio urbano y rural, o del uso del suelo, supuestos que difieren del presente [...] porque el plan no necesita su desarrollo a través de proyectos. $\mathrm{Y}$ en el otro, de admitirse que la revisión del Plan impugnado se hace a través de propuestas que se suceden en el tiempo, y en la última estaba vigente la nueva ley que establece este trámite, tampoco resultaría preceptivo, porque igualmente lo establece para proyectos sobre las mismas materias que el anterior, añadiendo que requieran una evaluación por afectar a espacios de la Red Natural 2000 en los términos previstos en la Ley 42/2007, de 13 de diciembre, de Patrimonio Natural y de la Diversidad [...] texto que condiciona la evaluación a las repercusiones en el espacio en perjuicio de su integridad. Consecuencias que analizando el contenido del Plan no se aprecian asociando la presencia del lobo en una parte del referido espacio².

Respecto a la razonabilidad y adecuación de las medidas de control de la especie, el Tribunal considera que las alegaciones de los recurrentes deben igualmente ser desestimadas, porque

a priori no resultan incompatibles con la conservación de la especie [...], si se tiene en cuenta que son muy limitados y con carácter excepcional los controles que periódicamente se fijan sin tener carácter cinegético, y que la especie se está recuperando y expandiendo su espacio [...] Aparte de que se trata de controles necesarios en la gestión de la naturaleza para resolver los conflictos

\footnotetext{
${ }^{2}$ Fundamento de Derecho quinto.
} 
de intereses existentes debido a los daños causados por los lobos a la cabaña ganadera en los últimos años al ser uno de los recursos básicos de alimentación de la especie, y ello sin perjuicio de que en el texto normativo que se revisa merece una mayor protección los intereses de la población rural al constituir las explotaciones ganaderas la fuente de sus ingresos. En todo caso, responde a la exigencia de conciliación eficaz de la protección de esta especie con los intereses de los ganaderos, que también merecen protección. No resta tampoco relevancia a la consideración precedente que el lobo cuente con el menor nivel de protección establecida por el Convenio Internacional de Berna mediante una reserva del Estado Español, y de las Directivas Europeas, y de que no se aprecie la falta de coordinación entre las Administraciones competentes 3 .

El Tribunal entiende no obstante que las cuestiones planteadas eran de complejidad fáctica y jurídica, y que estaban en litigio intereses públicos de protección de la naturaleza, por lo que no impone condena en costas.

El segundo de los recursos referidos (441/2016) ha sido igualmente desestimado, y esta vez con condena en costas, por la Sentencia 517/2017, de 12 de junio. Los recurrentes alegaban también en este caso defectos de tramitación y arbitrariedad del Programa anual de control de la especie del año 2015. El Letrado de la Administración entendió frente a ello que se había producido la pérdida sobrevenida del objeto del proceso, pues la resolución impugnada tenía una vigencia de un año y al formular la contestación a la demanda en el mismo mes de la fecha de la resolución, pero en 2016, el recurso devenía innecesario. El Tribunal no aceptó el argumento, por entender que los sucesivos Planes o Programas de Actuación de Control del Lobo no derogan ni dejan sin efecto los anteriores ${ }^{4}$.

En cuestiones formales, el Tribunal entiende que aunque existieron algunos defectos en la tramitación del Programa (notablemente falta de publicación en el BOPA), estos no deben reputarse invalidantes, pues ninguna indefensión ha

\footnotetext{
${ }^{3}$ Fundamento de Derecho cuarto.

${ }^{4}$ Fundamento de Derecho segundo.
} 
ocasionado a las entidades recurrentes, que son plenamente conocedoras de la misma, como pone de manifiesto su impugnación. También se niega el argumento de falta de motivación por entender que había suficientes estudios de diagnosis de la situación del lobo para avalar el Programa aprobado5.

Sobre el fondo del asunto, se valida la relación del Programa aquí impugnado con el II Plan del Lobo y se niega una posible vulneración del Reglamento de Caza del Principado de Asturias (Decreto 24/1991, de 7 de febrero). Los recurrentes sostenían que la posibilidad de autorizar batidas de lobos en el marco de cacerías de otras especies legalmente autorizadas, suponía convertir de facto al lobo en una especie cinegética, pese a no estar incluido en el Reglamento de Caza. El Tribunal parece atenerse en este punto a una perspectiva dogmática, negando la invocabilidad del Reglamento de Caza por ser precisamente el lobo una especie no cinegética. Digo "parece" porque realmente la redacción del pasaje resulta desafortunada, arrastrando alguna errata u omisión, como puede verse a partir de la transcripción literal del texto de la sentencia disponible en la base de datos de sentencias del CGPJ:

A ello ha que decir que aunque se empleen técnicas similares a la caza, el lobo no tiene la consideración de especie cinegética, por lo que le es ( $\mathrm{sic}$ ) de aplicación el referido Decreto, de forma que no cabe vulneración alguna del mismo ${ }^{6}$.

\section{Otro episodio judicial en torno al nuevo-viejo plan estratégico de residuos}

La STJA 500/2017, de 5 de junio, ha resuelto el recurso 367/2016 presentado por dos asociaciones ecologistas contra el Acuerdo del Consejo de Gobierno del Principado de Asturias de fecha 17 de febrero de 2016, por el que se aprobó el Plan Estratégico de Residuos del Principado de Asturias 2014-2024 y contra la Resolución de 1 de marzo de 2016 de la Consejería de Infraestructuras, Ordenación del Territorio y Medio Ambiente que ordenó la publicación en el BOPA del indicado Plan Estratégico (PERPA). Es el Plan que

\footnotetext{
${ }^{5}$ Fundamento de Derecho quinto.

${ }^{6}$ Fundamento de Derecho sexto.
} 
ha comenzado ya a revisarse conforme se ha comentado en la sección de derecho y normativa ambiental asturiana.

La resolución impugnada se dictó en su momento para cumplir con las SSTJA $544 / 2015$ y 584/2015, de 6 y el 20 de julio de 2015, respectivamente, que habían anulado la versión del PERPA aprobado en 2012 por razones de falta de publicidad. En concreto la resolución acordó la información pública de una versión adaptada del documento del Plan anulado, versión que finalmente constituye el Plan aprobado por el Consejo de Gobierno en 2016. Lo que los recurrentes aducen como motivo de nulidad, o en su defecto anulabilidad, es precisamente que lo que se publicó en el trámite de información pública no fue el documento de evaluación ambiental del nuevo Plan, sino la evaluación ambiental del Plan originario anulado. Entre los dos textos había una modificación sustancial: la eliminación de una planta incineradora de residuos domésticos.

Lo que el Tribunal concluye es que el cumplimiento de sus sentencias de 2015 no obligaba a efectuar de nuevo un estudio de impacto ambiental, toda vez que la eliminación de la planta incineradora en ningún caso supone un empeoramiento del medio ambiente. Por lo tanto la evaluación ambiental del primer documento puede aplicarse también al segundo, dado que preveía las consecuencias que para el medio ambiente pudieran ocasionar el resto de medidas a adoptar con el Plan (medidas subsistentes). En concreto se dispone que

tenemos que entender que la modificación del Plan aprobado, suprimiendo del mismo la instalación de una planta de tratamiento de basura doméstica, no requería la tramitación de un nuevo estudio de impacto ambiental del que tan solo se precisa excluir las consecuencias que para el medio ambiente pudieran suponer la instalación de la indicada planta?

En relación con la normativa aplicable, el Tribunal aprecia que sus sentencias de 2015 obligaban a retrotraer el expediente inicial, y por tanto la normativa

\footnotetext{
${ }^{7}$ Fundamento de Derecho segundo.
} 
aplicable ha de ser la que estaba vigente en el momento en que aquel se inició, no en el de elaboración del nuevo Plan. Se dispone así en efecto que

entendemos que la Ley 21/2013, de 9 de diciembre, de evaluación ambiental, que deroga la ley 9/2006, de 28 de abril, sobre evaluación de los efectos de determinados planes y programas en el medio ambiente, según su Disposición Derogatoria Única, con efectos a partir de un año de su entrada en vigor, 11 de diciembre de 2014, no es aplicable al caso de autos toda vez que el inicio del expediente al que se retrotraen las actuaciones es anterior a la indicada fecha8.

Además, los recurrentes alegaban que no se había cumplido una obligación esencial: dar publicidad al Plan en su integridad. En este punto el Tribunal entiende que publicar la modificación es en este caso suficiente, pues

en cuanto que se trata de excluir de la evaluación ambiental el impacto que sobre el medio ambiente pudiera producir la planta que se excluye del Plan Estratégico inicial [...] no puede producir su exclusión ningún impacto ambiental nuevo que la eliminación de los estimados por la instalación de la planta ${ }^{9}$.

E igualmente se señala que

la publicación del Plan originario y la Memoria que lo modifica, se viene a integrar en el indicado Plan, modificándolo y eliminando el impacto ambiental que pudieran producir los elementos sacados del referido Plan, cumpliendo con lo acordado en las sentencias que lo anulaban, dejadas firmes, en las que se ponía de manifiesto la necesidad de dar publicidad a la modificación efectuada en el Plan Estratégico ${ }^{10}$.

Finalmente, los recurrentes habían atacado la discrecionalidad de la Administración en la configuración de las medidas adoptadas para lograr los objetivos previstos por el artículo 22 de la Ley 22/2011, de 28 de julio, de residuos y suelos contaminados para el horizonte 2020. El argumento

\footnotetext{
${ }^{8}$ Fundamento de Derecho tercero.

${ }^{9}$ Fundamento de Derecho tercero.

${ }^{10}$ Fundamento de Derecho cuarto.
} 
sostenido era que tales medidas vulneraban el principio de jerarquía del mismo texto normativo. En este punto el Tribunal entiende que el PERPA contiene las medidas que la Administración autonómica ha decidido adoptar, y aplicando una suerte de teoría que respecto a ellas es

susceptible de denuncia su incumplimiento, tanto por el Gobierno de la Nación, como por quien ostente un interés legítimo, pero sin alcanzar a la impugnación de las medidas establecidas en el Plan para lograr los objetivos previstos, dado que obedece a la propia Administración fijarlos en uno u otro sentido dentro del Plan por ella establecido ${ }^{11}$.

Y por todo ello se desestima el recurso, con la imposición de costas por entender que los motivos de impugnación obedecían más simples formalidades que a razones reales, si bien las limita a 1.000 euros, por todos los conceptos, IVA incluido.

\section{Tributos, medio ambiente y redes de telecomunicaciones}

En sentencia 319/2017, de 17 de abril, el Tribunal Superior de Justicia desestima un nuevo recurso (el 60/2016) presentado por Telefónica Móviles España, S.A.U. contra una liquidación del Impuesto sobre Desarrollo de Determinadas Actividades que Inciden en el Medio Ambiente. En síntesis y salvo en lo que justamente a continuación se comentará, la parte actora basa su impugnación en los mismos motivos que habían fundado los recursos por ella presentados en 2014 y que habían sido ya desestimados en sentencias $781 / 2015$, de 3 de noviembre y 156/2016, de 7 de marzo ${ }^{12}$.

Los únicos argumentos nuevos, que son considerados pero descartados expresamente por la Sala, se refieren a una presunta falta de conformidad del gravamen con los arts. 12, 6.1 y "Anexo A" en relación con el art. 4 de la Directiva 2002/20/CE del Parlamento Europeo y del Consejo, de 7 de marzo de 2002, relativa a la autorización de redes y servicios de comunicaciones electrónicas y a la vulneración de los arts. 16, 17, 20 y 21 de la Carta de los Derechos Fundamentales de la Unión Europea.

\footnotetext{
${ }^{11}$ Fundamento de Derecho sexto.

${ }^{12}$ Cfr. crónica de esta revista correspondiente al primer trimestre de 2016, en el vol. VII, n. 1.
} 
En relación con estas supuestas infracciones, el TSJA aplica al impuesto aquí considerado la doctrina sentada por el TJUE en sentencia de 17 de diciembre de 2015 (asunto C-454/13) por lo que entiende que la conocida como Directiva de autorización no es de aplicación por falta de vinculación del hecho imponible al procedimiento de autorización general ${ }^{13}$. Igualmente descarta que quepa hablar de vulneración de la libertad de establecimiento

por encontrarnos ante la denominada Fiscalidad Asimétrica, en cuyo caso los impuestos deben valorarse teniendo en cuenta la relación competitiva de los afectados o no afectados por el tributo en cuestión, su finalidad y sus efectos, parámetros estos que en este caso están claramente definidos y excluyen por sí mismos la posibilidad de obstaculizar al libre establecimiento de otras empresas que, a mayor abundamiento, deberían pertenecer al mismo sector y operar en el mismo mercado (Asunto G-409/00, España contra Comisión).

Finalmente, el Tribunal tampoco acepta que pudiera considerarse vulnerado el principio "quien contamina paga" porque resulta admitida y clara

la afectación medioambiental de la telefonía, tal y como se desprende de la jurisprudencia existente respecto a la afectación de la salud por las ondas electromagnéticas e, incluso, corrobora lo dispuesto en el Real Decreto 1066/2001 al exigir medidas al amparo del principio de precaución por razones sanitarias, al amparo del art. 149.1 de la $C E^{14}$.

\footnotetext{
${ }^{13}$ Fundamento de Derecho cuarto.

14 Ídem.
} 